\title{
THE RELATIVE FIRE-RISK OF OIL AND AIR-BLAST TRANSFORMERS.
}

BY E. W. RICE, JR.

Two types of transformers have been extensively used in electrical installations up to date, distinguished by the methou of insulation and cooling employed. The "oil transformer" relies upon oil as the cooling and insulating fluid. The "airblast transformer" contains insulation material mainly of cloth, paper, and wood impregnated with oil or varnish, and is cooled by the circulation of a blast of air. In both types the insulating material is of an inflammable nature, and under certain abnormal conditions may take fire with more or less serious consequences.

The electrical engineer must, therefore, consider carefully not only the relative but the actual fire-hazard which exists, and by proper and common-sense methods minimize such danger. Both types can be made entirely safe by correct methods of design and installation.

I think it will be admitted that in general that type which contains the greater quantity of inflammable material will occasion the greater fire-hazard. The inflammable material in an air-blast transformer of say $1000-\mathrm{kw}$. capacity will amount to about $800 \mathrm{lb}$; in an oil-cooled transformer of the same capacity the amount will be about $7300 \mathrm{lb}$. While this comparison cannot be taken as a measure of the relative fire-risk, it is an indication to be considered, especially in view of the fluidity, the low temperature of ignition, and high calorific value of oil.

While the quantity of inflammable material in an air-blast transformer is, as stated, relatively small, it has an extended surface exposed to a large volume of air, and therefore, if a fire 
starts from internal causes, such as short circuit or extreme overload, is capable of rapid combustion. This combustion could be checked by shutting off the flow of air to a transformer by means of a diaphragm automatically closed by the melting of a. fusible link, the fusible link so located as to be melted by the first contact with flame; a method similar to that employed for closing fire-doors in buildings.

An oil transformer properly cooled is probably not particularly subject to ignition of the oil from internal burn-outs or arcs. It is well known that oil is an excellent medium for the smothering of alternating arcs, and this principle is utilized in connection with oil-switches. The vapor above the oil, may however, be ignited by electrical discharges. Even in this case, while the quantity of combustible material is enormous, the surface exposed is relatively small. The principle fire-hazard in an oil transformer is due to the large mass of inflammable liquid material which undercertain conditions may become totally consumed. It becomes a special hazard in the case of fire from sources external to itself.

Considerations of first cost, economy of space, simplicity, operating costs, etc., have resulted in placing transformers in the same room with switchboards and other apparatus, such as synchronous converters, motor-generators, etc. Under such conditions, it would seem that the air-blast transformer constituted the lesser fire-risk than the oil transformer, and would therefore be generally employed if the fire-risk were the only consideration. The air-blast type, however, is limited in practice to pressures of about 30000 to 35000 , as the static discharge which occurs at much higher pressures would in time break down the insulation. It is therefore necessary to employ oil insulation on the higher pressures now common.

The fire-risk can be practically eliminated by placing such. transformers in a room or rooms separated by suitable fire walls. from the other part of the plant. This plan has already been proposed and introduced. An entirely separate building, subdivided again into suitable rooms, may be employed where the maximum of safety is demanded. Much may be done to limit. the risk, even when the transformers are placed in the same room with other apparatus, by proper systems of piping for draining the oil away from the building, by placing the transformers in a depressed area of concrete arranged for rapid drainage, etc. Of course any of the methods commonly employed for preventing, 
limiting, or extinguishing oil.fires may properly be employed.

In closing I wish to state that I consider a discussion of this subject both timely and important. It is well for engineers to consider carefully the dangers of all kinds, both to life and to property, that may exist in connection with the use of electrical appliances. The art is not advanced by ignoring or belittling the existence of real difficulties, but rather by intelligently facing the problems which occur and seeking a proper solution. Electrical energy is capable of being produced, handled, and transmitted more safely than any other form of energy, and such dangers as exist usually can be foreseen and safe remedies can be applied. On the other hand, we must not exaggerate the danger of fire from the use of transformers of either the oil or airblast type. I believe the fire-hazard is extremely small, and can be and is being reduced to a negligible quantity by the adoption of methods similar to those I have outlined here. 